\title{
RECONSTRUCTION OF HYPERTREES
}

\author{
H. G. SENGE \\ In Memory of Ernst G. Straus
}

\begin{abstract}
In this paper we show that with essentially only one exception, every hypertree can be reconstructed from the set of maximal partial hypertrees $\{T-E \mid E \cap C \neq \varnothing\}$. The exceptional hypertrees are hyperchains of odd length $\left\{x_{0}, E_{1}, x_{1}, \ldots, E_{x}, x_{s}\right\}$, where

$$
\left|E_{1}\right|=\left|E_{3}\right|=\left|E_{5}\right|=\cdots \neq\left|E_{2}\right|=\left|E_{4}\right|=\left|E_{6}\right|=\cdots \text {. }
$$
\end{abstract}

1. Introduction. Let $G$ denote a graph with vertex-set $X=$ $\left\{x_{1}, \ldots, x_{n}\right\}$, and let $G_{i}$ denote the subgraph of $G$ that is generated by all the vertices of $G$ except $x_{i}$. In [6], Kelly proved that if $T$ and $T^{\prime}$ are trees with the same number of vertices, and if $T_{i} \cong T_{i}^{\prime}$ for all $i=1,2, \ldots, n$, then $T \cong T^{\prime}$. This gave rise to the Kelly-Ulam conjecture [6,7]:

Conjecture 1. Let $G$ and $G^{\prime}$ be graphs with the same number of vertices. If for some ordering of the vertices of $G$ and $G^{\prime}, G_{i} \cong G_{i}^{\prime}$ for all $i=1,2, \ldots$, then $G \cong G^{\prime}$.

Harary [4] reformulated the Kelly-Ulam Conjecture as follows:

Conjecture 2. Every graph $G$ is uniquely determined by the set of maximal subgraphs $\left\{G_{x} \mid x \in X\right\}$.

He called graphs for which Conjecture 2 is true, reconstructible graphs. In the same paper he also conjectured that every graph $G$ is reconstructible from the partial graphs $G_{e}$ that are obtained from $G$ by deleting an edge from $G$. Let us restate this as:

Conjecture 3. Every graph is uniquely determined by the set of maximal partial graphs $\left\{G_{e} \mid e \in \mathscr{E}\right\}$.

Harary and Palmer [5] extended Kelly's theorem by proving that every tree $T$ can be reconstructed from the set of maximal subtrees $T_{x}$, $\operatorname{deg} x=1$. Later, Bondy [2] extended Harary and Palmer's theorem by considering only those maximal subtrees $T_{x}$ where $x$ lies on the circumference of $T$. 
In this paper we present a reconstruction theorem for hypertrees that contains all the results mentioned above as special cases. Even though the transition from trees to hypertrees is not particularly difficult, we will present a complete proof of the reconstruction theorem for hypertrees that is considerably different from the proof given by Bondy.

2. Definitions. Let $X$ be a finite non-empty set, and let $\mathscr{E}$ be an indexed family of non-empty subsets of $X . H=(X, \mathscr{E})$ is called a hypergraph if $\bigcup_{E \in \mathscr{E}} E=X$. The elements of $X$ are called the vertices of $H$, and the elements of $\mathscr{E}$ are called the edges of $H$. If the elements of $\mathscr{E}$ are distinct, so that $\mathscr{E}$ is a set of subsets of $X$, then $H$ is called a simple hypergraph.

Let $X^{\prime} \subseteq X$ and let $\mathscr{E}^{\prime}=\left\{E \cap X \mid E \in \mathscr{E}\right.$ and $\left.E \cap X^{\prime} \neq \varnothing\right\}$. The hypergraph of $H^{\prime}=\left(X^{\prime}, \mathscr{E}^{\prime}\right)$ is called the subhypergraph that is generated by $X^{\prime}$. If $X^{\prime}=X-\{x\}$, then $H^{\prime}$ is called a maximal subhypergraph of $H$, and is denoted by $H-x$.

Let $\mathscr{E}^{\prime} \subseteq \mathscr{E}$ and let $X^{\prime}=\cup_{E \in \mathscr{E}^{\prime}} E$. The hypergraph $H^{\prime}=\left(X^{\prime}, \mathscr{E}^{\prime}\right)$ is called the partial hypergraph of $H$ that is generated by $\mathscr{E}^{\prime}$. If $\mathscr{E}^{\prime}=\mathscr{E}-\{E\}$, then $H^{\prime}$ is called a maximal partial hypergraph of $H$, and is denoted by $H-E$.

A hypergraph $H$ that is uniquely determined by the set of maximal subhypergraphs $\{H-x \mid x \in X\}$, is said to be vertex-reconstructible. A hypergraph $H$ that is uniquely determined by the set of maximal partial hypergraphs $\{H-E \mid E \in \mathscr{E}\}$, is said to be edge-reconstructible.

The incidence matrix of a hypergraph $H$ with $n$ vertices $x_{1}, \ldots, x_{n}$, and $m$ edges $E_{1}, \ldots, E_{m}$, is the matrix $A=\left(a_{i j}\right)$, where

$$
a_{i j}= \begin{cases}1 & \text { if } x_{j} \in E, \\ 0 & \text { if } x_{j} \notin E_{i} .\end{cases}
$$

For every hypergraph $H$, there exists a hypergraph $H^{*}$, whose incidence matrix is the transpose of the incidence matrix of $H . H^{*}$ is called the dual hypergraph of $H$. Let us denote the vertices of $H^{*}$ by $x_{1}^{*}, \ldots, x_{m}^{*}$, and the edges of $H^{*}$ by $E_{1}^{*}, \ldots, E_{n}^{*}$.

Proposition 1. A hypergraph $H$ is $\left\{\begin{array}{l}\text { vertex } \\ \text { edge }\end{array}\right\}$-reconstructible if and only if $H^{*}$ is $\left\{\begin{array}{c}\text { edge } \\ \text { vertex }\end{array}\right\}$-reconstructible.

Proof. $\left(H-x_{i}\right)^{*}=H^{*}-E_{i}^{*}$ for all $i=1, \ldots, n$, and $\left(H-E_{j}\right)^{*}=$ $H^{*}-x_{j}^{*}$ for all $j=1, \ldots, m$. This follows immediately by considering the respective incidence matrices. 
Proposition 2. Let $X$ be a finite set of $n$ elements, and let $\mathscr{E}_{1}=\{A \subseteq$ $X|| A \mid$ is odd $\}$, and $\mathscr{E}_{2}=\{A \subseteq X|| A \mid$ is even, and $\neq 0\}$. If $H_{1}=\left(X, \mathscr{E}_{1}\right)$ and $H_{2}=\left(X, \mathscr{E}_{2}\right)$, then $H_{1}-x=H_{2}-x$ for all $x \in X$.

Proof. For all $x \in X, H_{i}-x$ is the complete hypergraph on $X+\{x\}$, for both $i=1$ and $i=2$.

From Propositions 1 and 2, it follows that in general, edge or vertex reconstruction of hypergraphs is impossible. However, it now becomes an interesting problem to determine all reconstructible hypergraphs. One large class of reconstructible hypergraphs that will be considered later is the class of hypertrees.

A hypergraph is said to be regular if all vertices have the same degree. If all edges contain the same number of vertices, then it is called a uniform hypergraph. Thus $H$ is uniform if and only if $H^{*}$ is regular.

A hyperchain of length $s$ is an alternating sequence, $\left\{x_{0}, E_{1}\right.$, $\left.x_{1}, \ldots, E_{s}, x_{s}\right\}$, of $s+1$ distinct vertices, and $s$ distinct edges, where $x_{i-1} \in E_{i}$ and $x_{i} \in E_{i}$ for all $i=1,2, \ldots, s$. An alternating sequence that satisfies all conditions for a hyperchain exception that $x_{0}=x_{s}$, is called a hypercycle. The distance between two vertices $x$ and $x^{\prime}$ is defined as the length of a shortest hyperchain that connects $x$ and $x^{\prime}$, and is denoted by $d\left(x, x^{\prime}\right)$. The eccentricity of a vertex $x \in X$ is defined as

$$
e(x)=\max _{x^{\prime} \in X} d\left(x, x^{\prime}\right) \text {. }
$$

The radius and diameter of a hypergraph can be defined in terms of the eccentricity of the vertices. Let

$$
r=\min _{x \in X} e(x)
$$

and

$$
d=\max _{x \in X} e(x) .
$$

The set of all vertices of a hypergraph $H$ that have minimal eccentricity is called the center of $H$, while the set of all vertices of maximal eccentricity is called the circumference of $H$. The center and the circumference of $H$ are denoted by $Z$ and $C$ respectively, where

$$
Z=\{x \in X \mid e(x)=r\},
$$

and

$$
C=\{x \in X \mid e(x)=d\} .
$$

A hyperchain of maximum length $d$ in a hypergraph $H$ is called a Diameter of $H$. [Note that the words "diameter" and "Diameter" have different meanings]. 
A vertex $x \in X$ is called a cut vertex of $H$ if $H-x$ is disconnected. A block is a connected hypergraph that has no cut vertices. The maximal sub-hypergraphs of $H$ that are blocks, are called the blocks of $H$.

LEMMA 1. A sub-hypergraph $B$ of a hypergraph $H$ is a block if and only if any two vertices of $B$ lie on a hypercycle in $B$.

LEMMA 2. The center of a hypergraph is contained in one of its blocks.

The proofs of these two lemmas are identical to the proofs of the corresponding lemmas for graphs and can be found elsewhere [1].

3. Hypertrees. A connected hypergraph that does not contain a hypercycle is called a hypertree. If two edges $E$ and $F$ of a hypergraph $H$ have at least two vertices $x$ and $y$ in common, then $H$ contains the hypercycle $\{x, E, y, F, x\}$. Consequently, any two edges of a hypertree have at most one common vertex.

For hypertrees, Lemmas 1 and 2 have the following implications: 1. The blocks of a hypertree are the edges, and 2. The center of a hypertree consists of a single vertex, or a single edge. Moreover, the type of center of a hypertree is completely determined by its diameter. Hypertrees with even diameter have a vertex as the center, while hypertrees with odd diameters have an entire edge as the center. From now on we will refer to a hypertree as being even or odd according to whether its diameter is even or odd.

4. Reconstruction of hypertrees. Let $z$ be the center of an even hypertree $T$. Let us decompose $T$ by separating all the edges that contain $z$. The resulting components are partial hypertrees of $T$, and are called the central branches of $T$. Thus every central branch of $T$ contains $z$ as a vertex of degree 1 . Let $Z$ be the center of an odd hypertree $T$. Then $T-Z$ consists of at most $|Z|$ components. Each of these components together with $Z$ is a partial hypertree of $T$, and is called a central branch of $T$. Thus every central branch of $T$ contains $Z$ as a terminal edge. A central branch of a hypertree $T$ that contains at least one vertex on the circumference of $T$ is called a radial branch. There is one basic property that distinguishes even and odd hypertrees: An even hypertree can have any number of central branches, while an odd hypertree can have at most $|Z|$ central branches. Before we launch into the proof of the reconstruction theorem, we must consider one exceptional case. 
LEMMA. Let $T$ be an even hypertree that consists of a single alternating hyperchain $E_{0}, E_{1}, \ldots, E_{s}$, where $E_{i-1} \cap E_{i} \neq \varnothing$, and $\left|E_{0}\right|=\left|E_{2}\right|=\left|E_{4}\right|=$ $\cdots \neq\left|E_{1}\right|=\left|E_{3}\right|=\left|E_{5}\right|=\cdots$. Let $T_{1}=T-E_{0}$ and $T_{2}=T-E_{s}$. Then $T_{1} \neq T_{2}$, and $T_{1}-E_{1} \cong T_{2}-E_{0} \cong T_{2}-E_{s-1}$.

Proof. Clear.

Let $T=(X, \mathscr{E})$ be a hypertree, let $C$ denote the circumference of $T$, and let $\mathscr{E}_{C}=\{E \in \mathscr{E} \mid E \cap C \neq \varnothing\}$. We can now prove the reconstruction theorem.

THEOREM 1. Every hypertree $T$, that is not an alternating hyperchain of odd length can be reconstructed from the set of maximal partial hypertrees $\left\{T-E \mid E \in \mathscr{E}_{C}\right\}$.

Proof. The proof will be divided into three parts:

1. $\left|\mathscr{E}_{C}\right|>2$, and $T-E$ is even (odd) for all $E \in \mathscr{E}_{C}$.

2. $\left|\mathscr{E}_{C}\right|>2$, and there exist hyperedges $E$ and $F \in \mathscr{F}_{C}$ so that $T-E$ is even, and $T-F$ is odd.

\section{3. $\left|\mathscr{E}_{C}\right|=2$.}

1. Let us first consider the case where $T-E$ is even for all $E \in \mathscr{E}_{C}$. Then $T$ must be even, and the center of $T$ is the same as the center of $T-E$ for all $E \in \mathscr{E}_{C}$. Let $b_{i}$ denote the number of radial branches of $T$ that have exactly $i$ edges in $\mathscr{E}_{C}$, and let $b_{0}$ denote the number of non-radial central branches of $T$. For every edge $E \in \mathscr{E}_{C}$, let $b_{i}(E), i \geq 0$, be defined as above for the hypertree $T-E$. Let $p(t)=b_{1}+b_{1} t+b_{2} t^{2}+\cdots$, and let $p_{E}(t)=b_{0}(E)+b_{1}(E) t+b_{2}(E) t^{2}+\cdots$. The polynomials $p_{E}(t)$, where $E \in \mathscr{E}_{C}$, are easily derived from the structure of $T-E$ and are closely related to $p(t)$. In particular, if an edge $E \in \mathscr{E}_{C}$ is part of a radial branch that has $i$ edges in $\mathscr{E}_{C}$, then

$$
b_{j}(E)=\left\{\begin{array}{l}
b_{j}+1, \quad \text { if } j=i-1, \\
b_{j}-1, \quad \text { if } j=i \\
b_{J}, \quad \text { if } j \neq i-1, \text { and } j \neq i
\end{array}\right.
$$

and hence

$$
p(t)-p_{E}(t)=t^{i}-t^{i-1} \text {. }
$$

Consequently, for any two distinct edges $E$ and $F \in \mathscr{E}_{C}$, there exist positive integers $i$ and $j$, so that

$$
p_{E}(t)-p_{F}(t)=t^{j}-t^{j-1}-t^{i}+t^{i-1} \text {. }
$$


We can assume that $j \geq i$. (If not, interchange $E$ and $F$.)

At this point we have to consider two different cases:

$$
\begin{gathered}
p_{E}(t)=p_{F}(t), \text { for all } E \text { and } F \in \mathscr{E}_{C} . \\
p_{E}(t) \neq p_{F}(t), \text { for at least two edges } E \text { and } F \in \mathscr{E}_{C} .
\end{gathered}
$$

(1.1). It follows from (1) and (2) that all radial branches of $T$ have the same number of edges in $\mathscr{E}_{C}$, so that $p(t)=b_{0}+b_{i} t^{i}$, and for all $E \in \mathscr{E}_{C}$, $p_{E}(t)=b_{0}+t^{i-1}+\left(b_{i}-1\right) t^{i}$. Let $s$ denote the number of radial branches of $T$. Then $\left|\mathscr{E}_{C}\right|=i s$, and the total number of radial branches in all sub-hypertrees $T-E$, where $E \in \mathscr{E}_{C}$, is $i s(s-1)$. Every radial branch of $T$ occurs among the radial branches of all $T-E$ exactly $i(s-1)$ times. Thus the multiplicity of a radial branch among all $T-E$ under consideration is $i(s-1)$ times its multiplicity in $T$. With this observation we can identify all radial branches of $T$. If $i>1$, then the non-radial central branches of $T$ are precisely the non-radial central branches of $T-E$, for any $E \in \mathscr{E}_{C}$. Finally suppose that $i=1$. Having determined the radial branches of $T$, we can identify the nonradial central branch of $T-E$ that resulted from deleting $E$ for any $E \in \mathscr{E}_{C}$. The remaining non-radial central branches of $T-E$ are the non-radial central branches of $T$.

(1.2). Choose $E$ and $F \in \mathscr{E}_{C}$ so that (2) is satisfied and $j>1$. Then all central branches of $T-E$ except those having $i$ or $i-1$ edges in $\mathscr{E}_{C}$ and all central branches of $T-F$ except those having $j$ or $j-1$ edges in $\mathscr{E}_{C}$ completely determine the central branches of $T$ unless $j-1=i$.

If $j-1=i$, then the radial branches with exactly $i$ edges in $\mathscr{E}_{C}$ are determined by an argument similar to the one in 1.1 applied to the set of all sub-hypertrees $T-F$ for which $p_{F}(t)=p_{E}(t)$. This concludes the proof of part 1 for even hypertrees.

If $T-E$ is odd for all $E \in \mathscr{E}_{C}$, then $T$ is odd and the center $Z$ of $T$ is the same as the center of $T-E$ for all $E \in \mathscr{E}_{C}$. Let us replace $Z$ in each partial hypertree $T-E$ by a star $S$ whose vertex-set is $Z \cup\left\{z_{0}\right\}$, and whose edge-set is $\left\{\left(z_{0}, z\right) \mid z \in Z\right\}$. The resulting hypertrees are all even, and are partial hypertrees of the hypertree $T^{\prime}$ that is obtained by replacing the center of $Z$ by the star $S$. By part 1 of this theorem for even hypertrees, $T^{\prime}$ can be reconstructed from the set of even partial hypertrees. Once $T^{\prime}$ is reconstructed, $T$ can be obtained by replacing the star $S$ in $T^{\prime}$ by $Z$.

2. In this case, either $T-E$ is the only partial hypertree that is even, or $T-F$ is the only partial hypertree that is odd. In either case, let $E_{1}$ 
denote the unique edge in $\mathscr{E}_{C}$ so that the parity of $T-E_{1}$ is unique among the partial hypertrees under consideration. Then $T$ has only two radial branches $B_{1}$ and $B_{2}$, where $B_{1}$ has one edge $E_{1} \in \mathscr{E}_{C}$, and $B_{2}$ has at least two edges in $\mathscr{E}_{C}$. For any edge $E \in \mathscr{E}_{C}, E \neq E_{1}$, the non-radial central branches of $T-E$ are the non-radial central branches of $T$. The radial branches of $T$ can be reconstructed with ease in all cases except those in which

$$
B_{2} \text { has exactly two edges } E_{2} \text { and } E_{2}^{\prime} \in \mathscr{E}_{C} \text {, }
$$

$$
\text { The circumference of } T-E_{1} \text { meets four edges and, }
$$

$$
T-E_{2} \cong T-E_{2}^{\prime} \text {. }
$$

In all other cases, one radial branch $B_{i}(i=1,2)$ of $T$ can be identified immediately, and this uniquely determines $B_{i}-E_{i}$. Now $B_{3-\imath}$ can be identified in $T-E_{i}$.

Suppose that $T$ satisfies (2.1)-(2.3). Under the isomorphism (2.3), the center and the radial branches of $T-E_{2}$ correspond to the center and the radial branches of $T-E_{2}^{\prime}$. This implies that

$$
B_{1} \cong B_{1} \quad \text { and } \quad B_{2}-E_{2} \cong B_{2}-E_{2}^{\prime},
$$

or

$$
B_{1} \cong B_{2}-E_{2}^{\prime} \text { and } B_{2}-E_{2} \cong B_{1} \text {. }
$$

In either case $B_{2}-E_{2} \cong B_{2}-E_{2}^{\prime}$. Without loss of generality we can assume that under the isomorphism (2.3), $E_{1}$ in $T-E_{2}$ is isomorphic to $E_{1}$ in $T-E_{2}^{\prime}$, and if we restrict the isomorphism (2.3) to $T-E_{1}$, then we see that

$$
\left(T-E_{1}\right)-E_{2} \cong\left(T-E_{1}\right)-E_{2}^{\prime} .
$$

Let $E_{1}^{\prime}$ and $E_{1}^{\prime \prime}$ denote the two hyperedges of $B_{1}-E_{1}$ that meet the circumference of $T-E_{1}$. If $\left(T-E_{1}\right)-E_{1}^{\prime} \not \equiv\left(T-E_{1}\right)-E_{1}^{\prime \prime}$, then $B_{2}$ can be identified in $T-E_{1}$. Therefore, let us suppose that in addition to (2.1)-(2.3), $T$ satisfies

$$
\left(T-E_{1}\right)-E_{1}^{\prime} \cong\left(T-E_{1}\right)-E_{1}^{\prime \prime} .
$$

If the two radial branches of $T-E_{1}$ are isomorphic, then $T$ can be reconstructed immediately by joining an appropriate edge to any one of the four edges that meet the circumference of $T-E_{1}$. The edge that must be added to $T-E_{1}$ can be determined by comparing the four edges mentioned above with the two edges that meet the circumference in $T-E_{2}$.

Now suppose that the radial branches of $T-E_{1}$ are not isomorphic. Let $D=\left\{E_{1}^{\prime}, F_{1}, F_{2}, \ldots, F_{s}, E_{2}^{\prime}\right\}$ denote the Diameter of $T-E_{2}$ that 
connects $E_{1}^{\prime}$ and $E_{2}^{\prime}$. $T-E_{2}$ can be derived from $T-E_{1}$ by joining $E_{1}$ to one end of $D$ and removing the edge that is isomorphic to the other end of $D$ from $T-E_{1}$. This can be done in two ways and leads to two hypertrees with unique Diameters

$$
\left\{E_{1}, E_{1}^{\prime}, F_{1}, F_{2}, \ldots, F_{s}, E_{2}^{\prime}\right\}
$$

and

$$
\left\{E_{1}^{\prime}, F_{1}, F_{2}, \ldots, F_{s}, E_{2}^{\prime}, E_{1}\right\} .
$$

If these two hypertrees are isomorphic, then their Diameters are also isomorphic. Hence $E_{1} \cong E_{2}^{\prime} \cong F_{1} \cong F_{2}-\cdots \cong E_{2}^{\prime} \cong E_{1}$, or $E_{1} \cong E_{1}, E_{1}^{\prime}$ $\cong E_{2}^{\prime}$, and $F_{\imath} \cong F_{s-1}$. The first case is impossible because it implies that the two hypertrees consist only of the Diameters. In the second case, the isomorphism between the two hyperetrees induces the isomorphism

$$
\left(\left(T-E_{1}\right)-E_{2}\right)-E_{2}^{\prime} \cong\left(\left(T-E_{1}\right)-E_{1}^{\prime \prime}\right)-E_{1}^{\prime} .
$$

But this in turn implies that $T-E_{1}$ has isomorphic radial branches which contradicts the first sentence of this paragraph. Thus there is only one way of transforming $T-E_{1}$ into $T-E_{2}$, and this leads to a unique reconstruction of $T$.

3. If $\left|\mathscr{E}_{C}\right|=2$, then $T$ has a unique Diameter. Let us denote the sequence of edges in the Diameter of $T$ by $\left\{E_{1}, E_{2}, \ldots, E_{d}\right\}$. If $T-E_{1}$ does not have a unique Diameter, then the radial branch of $T$ that contains $E_{d}$ can be identified in $T-E_{1}$, and then the radial branch that contains $E_{1}$ can be identified in $T-E_{d}$. This determines the edge $E_{1}$, and now $T$ can be reconstructed directly from $T-E_{1}$.

Now suppose that $T-E_{1}$ and $T-E_{d}$ have unique Diameters. $T$ can be reconstructed by superimposing the two maximal hypertrees in such a way that the two Diameters together yield the Diameter of $T$. This can be done in at most four ways.

$$
\begin{gathered}
E_{1}, E_{2}, \ldots, E_{d-1} \\
E_{2}, \ldots, E_{d-1}, E_{d} \\
E_{1}, \ldots, E_{d-2}, E_{d-1} \\
E_{2}, E_{3}, \ldots, E_{d} \\
E_{1}, E_{2}, \ldots, E_{d-1} \\
E_{d}, \ldots, E_{3}, E_{2} \\
E_{1}, \ldots, E_{d-2}, E_{d-1} \\
E_{d}, E_{d-1}, \ldots, E_{2} .
\end{gathered}
$$


One of these superpositions is always possible. Let us assume that the Diameter of the two maximal sub-hypertrees are labeled so that one superposition is represented by (3.1). If the two hypertrees can also be superimposed as indicated by (3.2), then $E_{1} \cong E_{3} \cong E_{5} \cong \cdots$, and $E_{1} \cong$ $E_{2} \cong E_{3} \cong \cdots$. Moreover, $T$ can have no side branches in this case, because $E_{2}$ and $E_{d-1}$ are connected to only two other edges. If $T$ is even, the terminal edges of both $T-E_{1}$ and $T-E_{d}$ are isomorphic. Thus both superpositions of $T-E_{1}$ and $T-E_{d}$ lead to the same result. If $T$ is odd, then $T-E_{1}=T-E_{d}$, and the terminal edges of $T-E_{1}$ need not be isomorphic. Thus the two superpositions of $T-E_{1}$ and $T-E_{d}$ will lead to different hypertrees if the terminal edges of $T-E_{1}$ are not isomorphic.

If the two hypertrees can be superimposed as indicated by (3.3), then $E_{i}=E_{d+2-i}, 1 \leq i \leq d$. this implies that there exists an automorphism of $T-E_{1}$ that reverses its Diameter, and therefore both superpositions lead to $T$.

If the two hypertrees can be superimposed as indicated by (3.4), then $E_{i}=E_{d-i}, 1 \leq i \leq d$. This implies that there exists an automorphism of $T-E_{d}$ that reverses its Diameter, and therefore both superpositions lead to $T$.

If the two hypertrees can be superimposed as indicated by (3.4), then $E_{i}=E_{d-\imath}, 1 \leq i \leq d$.This implies that there exists an automorphism of $T-E_{d}$ that reverses its Diameter, and therefore both superpositions lead to $T$.

5. Concluding remarks. It should be clear that vertex-reconstruction for hypergraphs is not a generalization of graph reconstruction in the sense of Conjecture 2, because the removal of a vertex from a hypergraph does not necessarily eliminate any edges. In fact, the edges of $H-x$ are obtained from the edges of $H$ by eliminating $x$ from the edges that contain it. On the other hand, edge-reconstruction of hypergraphs is a generalization of graph reconstruction in the sense of Conjecture 3, provided we ignore isolated vertices.

Reconstruction in the sense of Conjecture 2 can be extended to hypergraphs as follows: $H=(X, \mathscr{E})$ be a hypergraph, and let $X_{x}=X-$ $\{x\}, \mathscr{E}_{x}=\{E \in \mathscr{E} \mid x \notin E\}$, and $H_{x}=\left(X_{x}, \mathscr{E}_{x}\right)$. The partial hypergraphs $H_{x}$ are maximal in the sense that $\mathscr{E}_{x}$ is the minimal subset of $\mathscr{E}$ whose elements don't contain $x$. Let us call a hypergraph $H$ strongly reconstructible if it is uniquely determined by its partial hypergraphs in $H_{x}$.

Finally we point out that, as for graphs, it can be shown that every regular hypergraph with at least four edges is edge-reconstructible, and 
consequently, every uniform hypergraph with at least four vertices is vertex-reconstructible. This leads us to suspect that the following conjecture is true:

Conjecture 4. Every uniform hypergraph of order $n \geq 4$, is strongly reconstructible.

\section{REFERENCES}

[1] M. Behzad and G. Chartrand, Introduction to the Theory of Graphs, Allyn and Bacon, Boston (1971).

[2] J. A. Bondy, On Kelly's congruence theorem for trees, Proc. Camb. Phil. Soc., 65 (1969), 387-397.

[3] C. Berge, Graphs and Hypergraphs, North Holland, Amsterdam (1973).

[4] F. Harary, On the Reconstruction of a Graph from a Collection of Subgraphs. Theory of Graphs and its Applications, Academic Press, New York (1964), 47-52.

[5] F. Harary and E. Palmer, The Reconstruction of a tree fromits maximal subtrees, Canad. J. Math., 18 (1966), 803-810.

[6] P. J. Kelly, A congruence theory for trees, Pacific J. Math., 7 (1957), 961-968.

[7] S. M. Ulam, A Collection of Mathematical Problems, Wiley, New York (1960).

Received June 7, 1984.

3437 Mountain View Ave.

LOS ANGELES, CA 90066 